\section{Electromyographic Analysis of Upper Limb and Hand Muscles during Horticultural Activity Motions}

\author{
Sin-Ae Park ${ }^{1}$, Sae-Room $\mathrm{Oh}^{2}$, Kwan-Suk Lee ${ }^{2}$, and Ki-Cheol Son ${ }^{1,3}$
}

ADDITIONAL INDEX WORDs. muscle activation, physical rehabilitation, gardening, horticultural therapy, human issues in horticulture

SUMMARY. This study used electromyographic analysis to investigate specific upper limb and hand muscle activation during 15 common horticultural activities. A total of 30 Korean adults between the ages of 20 and 30 years, with an average age of 24.8 years, were recruited from Konkuk University, Seoul, South Korea. Electromyographic measurements were made using a portable four-channel electromyograph. Bipolar surface electromyography (EMG) electrodes were attached to six upper limb muscles (i.e., upper trapezius, triceps-long head, biceps brachialis, flexor carpi ulnaris, flexor carpi radialis, and brachioradialis) and two hand muscles (i.e., thenar eminence and hypothenar eminence) on the dominant hand. These eight muscles that were selected play a major role in the operation of upper limbs and hand muscles for upper body low-impact activities. Each participant did the $\mathbf{1 5}$ horticultural activities on one occasion with two separate sessions. Each activity was performed for 60 seconds followed by a 15-second rest period sitting at a table on a height-adjusted chair between each activity. All eight muscles measured were used together during most of 15 horticultural activities. Upper trapezius, thenar eminence, and hypothenar eminence had higher muscle activity than the other muscles. Triceps-long head displayed very low EMG values compared with the other muscles. The EMG data will facilitate developing scientific and research-based gardening intervention and/or horticultural therapy programs for improving physical health and physical rehabilitation.

$\mathrm{P}$ articipation in horticultural activities has significant human health benefits. For example, lower cholesterol and blood pressure (Armstrong, 2000), better balance and gait speed (Chen and Janke, 2012), better hand functional ability (Park et al., 2009), fewer chronic conditions and functional limitations (Chen and Janke, 2012), improved overall physical health (Park et al., 2009), lower stress (Hawkins et al., 2011; Van Den Berg and Custers, 2011), better life satisfaction (Sommerfeld et al., 2010), and improved psychological well-being and social integration (Waliczek et al., 2005; Walsh et al., 2001) have each been attributed to horticultural activities.

Various horticultural activities involve physical exercise. Most gardening activities were determined as low to moderate physical activities in previous studies (Ainsworth et al., 2011;

This article was supported by Konkuk University in 2012 .

${ }^{1}$ Department of Environmental Sciences, Konkuk University, Seoul 143-701, Korea

${ }^{2}$ Department of Industrial Engineering, Hongik University, Seoul 121-791, Korea

${ }^{3}$ Corresponding author. E-mail: kcson@konkuk.ac.kr.
Gunn et al., 2005; Park et al., 2008a, 2008b, 2011, 2012). Ainsworth et al. (2011) reported 53 common gardening tasks as low to moderate in physical intensity in adults aged 25 to 65 years based on published data or estimates by experts on physical activity. Park et al. (2008a, 2011) reported that gardening activities using both the upper and the lower body (e.g., digging, weeding) were moderate in intensity while activities that mainly used the upper body (e.g., mixing soil, planting seedlings) involved lowintensity physical activity in adults over 65 years old. Horticultural activities such as propagation (cutting herbs) or planting transplants that used mainly the upper body while standing involved low-intensity physical activity, while making a vegetable garden combined both upper and lower body muscles at a moderate intensity (Park et al., 2012).

Moreover, weight-bearing motions are body movements that work against gravity. Weight-bearing exercise improves muscle strength and physical functional ability (Olivetti et al., 2007). Exterior stress such as high-intensity exercises that require mechanical load stimulates new bone formation (Turner and Robling, 2003). Weight-bearing gardening motions (e.g., digging, weeding) can improve muscle strength and bone mineral density (Restuccio, 1992; Turner et al., 2002).

Electromyography detects the electrical signal produced by muscle cells when these cells are electrically or neurologically activated. EMG has been used to investigate muscular activity, and the signals can be analyzed to detect medical abnormalities and activation level, or to analyze the biomechanics of human or animal movement (Bolgla and Uhl, 2007; De Luca, 1997). In a previous study, muscle activation for the motions of flower arrangements such as cutting, bending, and winding were analyzed by EMG (Lee et al., 2012). Although horticultural activities have been used for improving physical rehabilitation of individuals with disabilities (Jung, 2005; Kim, 2001), there is little EMG information on various horticultural activities that could potentially be used to maximize the positive effects of therapy. As a consequence, this study investigated specific muscles that play a major role in the operation of upper limbs and hand muscles for upper body low-impact activities and their activation using EMG measurements during 15 common horticultural activities in adults.

\section{Materials and methods}

Participants. A total of 30 adults between the ages of 20 and 30 years ( 20 males, 10 females) from Konkuk University, Seoul, South Korea, volunteered to participate in

\begin{tabular}{llll}
\hline $\begin{array}{l}\text { Units } \\
\begin{array}{l}\text { To convert U.S. to SI, } \\
\text { multiply by }\end{array}\end{array}$ & U.S. unit & SI unit & $\begin{array}{l}\text { To convert SI to U.S., } \\
\text { multiply by }\end{array}$ \\
\hline 3.7854 & gal & $\mathrm{L}$ & 0.2642 \\
2.54 & inch $(\mathrm{es})$ & $\mathrm{cm}$ & 0.3937 \\
25.4 & inch $(\mathrm{es})$ & $\mathrm{mm}$ & 0.0394 \\
0.4536 & $\mathrm{lb}$ & $\mathrm{kg}$ & 2.2046 \\
$\left({ }^{\circ} \mathrm{F}-32\right) \div 1.8$ & ${ }^{\circ} \mathrm{F}$ & ${ }^{\circ} \mathrm{C}$ & $\left({ }^{\circ} \mathrm{C} \times 1.8\right)+32$
\end{tabular}


the study. Criteria for participation were age and the absence of exercise for the muscles of the upper limbs $24 \mathrm{~h}$ before testing. The participants were provided descriptive information for all procedures to be used in the study. Demographic and health information (e.g., age, gender, dominant hand, experiences of neurologic diagnosis, pain in upper limbs or hands during the past six months) were collected. The average age of the participants was $( \pm$ SD $) 24.8 \pm 2.8$ years. All of the participants were righthanded, and no one had experienced any neurological problems or pain in the upper limbs or hands during the past six months. The mean height and body weight of the participants were $171.1 \pm 8.6 \mathrm{~cm}$ measured by anthropometer (model ok7979; Samhwa, Seoul, South Korea) and $70.3 \pm 14.9$ $\mathrm{kg}$ measured by body weight analyzer (model B5010; Shenzhen Simple Electronics, Shenzhen, China), respectively. A gift card was provided as an incentive for each participant at the completion of the test.

Horticultural activities. Fifteen common horticultural activities using primarily upper limbs and hand muscles were performed by the 30 participants in a glass greenhouse on the Konkuk University campus. The participants did each horticultural activity for $60 \mathrm{~s}$ (see Table 1 for detail descriptions) followed by a 15-s rest between each activity while sitting at a table on a height-adjustable chair to reduce noise for EMG value by unstable shoulder position. The participants were required to repeat the same motions for the each activity during the testing time. In our preliminary test, a 60 -s activity time and a 15-s resting period were found to be sufficient for the electromyographic analysis because EMG values of one

Table 1. Descriptions of $\mathbf{1 5}$ horticultural activities for electromyographic analysis of upper limb and hand muscles during horticultural activity motions.

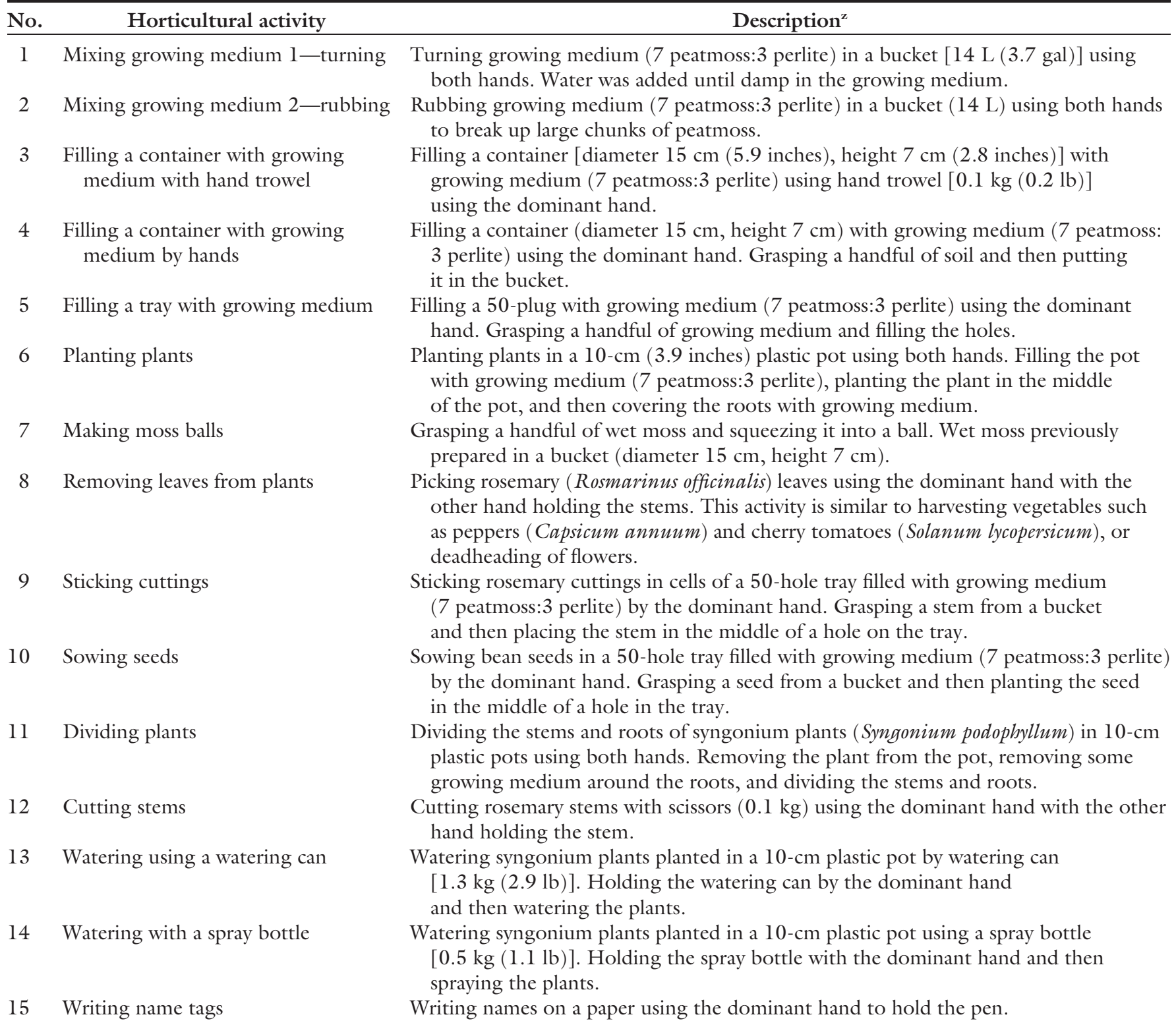

${ }^{\mathrm{z}}$ All participants sat on a height-adjusted chair and all horticultural activity materials were previously prepared on a table. The participants were required to repeat the same motions for the each activity for $60 \mathrm{~s}$ and then had a 15 -s rest while sitting on the chair between each activity. 
activity for $60 \mathrm{~s}$ maintained a certain range and a 15-s resting period allowed the values to revert back to an initial value. The activities were randomized for each participant. Participants wore a short-sleeve shirt to allow attachment of the EMG patches on their upper limb and gloves to protect the patches on the hands. The average temperature in the glasshouse was $( \pm \mathrm{SD})$ $25.2 \pm 4.9^{\circ} \mathrm{C}$ and the relative humidity was $38.0 \% \pm 26.2 \%$ (Acuba CS-201 digital hygro-thermometer; Chosun, Guangdong, China). These environmental conditions were appropriate for the research conducted in this study.

Measurements. A portable fourchannel EMG instrument (Myotrace 400; Noraxon, Scottsdale, AZ) was used. Bipolar surface EMG electrodes [silver/silver chloride $(\mathrm{Ag} / \mathrm{AgCl})$, $8-\mathrm{mm}$ radius] were attached to six upper limb muscles (i.e., upper trapezius, triceps-long head, biceps brachialis, flexor carpi ulnaris, flexor carpi radialis, and brachioradialis) and two hand muscles (i.e., thenar eminence and hypothenar eminence) on the dominant hand (Fig. 1). These eight muscles, which were selected by

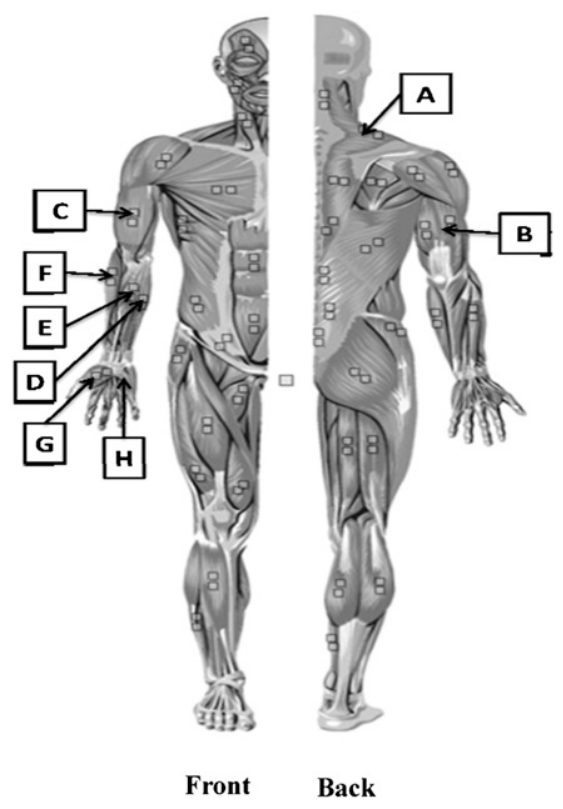

Fig. 1. Names of upper limb and hand muscles tested for electromyographic analysis during 15 horticultural activity motions: (A) upper trapezius, (B) triceps-long head, (C) biceps brachialis, (D) flexor carpi ulnaris, (E) flexor carpi radialis, (F) brachioradialis, (G) thenar eminence, $(\mathrm{H})$ hypothenar eminence (MyoResearch XP Clinical Edition 1.07 software; Noraxon, Scottsdale, AZ). the experts in laboratory of biomechanics, Department of Industrial Engineering, Hongik University, play a major role in the operation of upper limbs and hand muscles for upper body low-impact activities. Disposable, self-adhesive pregelled $\mathrm{Ag} / \mathrm{AgCl}$ dual electrodes were positioned parallel to and over the center of each muscle (Fujii and Moritani, 2012; Hawkes et al., 2011). The interelectrode distance (center to center) was $1 \mathrm{~cm}$ (Hong and Kim, 2009). To reduce interelectrode resistance, the surface of the skin was treated with alcohol before the electrodes were attached (Fujii and Moritani, 2012; Hawkes et al., 2011). Bipolar disposable hook wire electrodes were used for the intramuscular recording of activity. Thin elastic nylon bandages were then used to firmly hold the lead lines to the body to prevent artifacts that might be caused by displacement of the electrodes.

The participants completed the 15 activities on one occasion with two separate sessions since only four muscle positions could be tested at the same time. The triceps-long head, flexor carpi radialis, thenar eminence, and hypothenar eminence were randomly measured during the first session, and the upper trapezius, biceps brachialis, flexor carpi ulnaris, and brachioradialis were tested in the second session after a 10 -min rest. Before starting the first activity in each session, the participants sat on a chair with their hands on their knees for $30 \mathrm{~s}$ to obtain a resting EMG measurement as an EMG baseline. A 30-s resting time was found to be sufficient for the electromyographic analysis in our preliminary testing.

Data Analysis. Descriptive information was handled using Excel (Office 2007; Microsoft Corp., Redmond, WA). MyoResearch XP Clinical Edition 1.07 software (Noraxon) was used for analyzing EMG data for the horticultural activities. The sampling frequency was $1000 \mathrm{~Hz}$ and the data for the first $3 \mathrm{~s}$ was deleted to compensate for the time required to start an activity. Duncan's multiple range tests at $P<0.05$ were used to compare means using SAS (version 9.3 for Windows; SAS Institute, Cary, NC).

\section{Results and discussion}

The participants used all eight muscles during the 15 horticultural activities although the degrees of usage varied among muscles (Table 2 ). Mixing soil involved two motions: turning soil in the bucket and rubbing soil by hand to break up clods (Table 1). The upper trapezius and biceps brachialis were mainly used for turning and rubbing soil by hand, whereas breaking up clods used more upper limbs and hand muscles than turning soil (Table 2).

Filling a bucket with growing medium and transplanting plants primarily used the upper trapezius, thenar eminence, and hypothenar eminence (Table 2). To fill a container or a tray with growing medium, the participants picked up the growing medium by hand or using a hand trowel and then transferred it into a container or tray from the bucket (Table 1). The hypothenar eminence and thenar eminence were significantly used during the gripping motions required for picking up the growing medium and the upper trapezius for transferring growing medium from the bucket to the tray. The muscles such as biceps brachialis and brachioradialis were significantly used during the task, filling a container with growing medium by hand trowel because of holding a weighty tool such as hand trowel (Table 2).

Planting involved two steps: partially filling the pot with growing medium and then adding additional growing medium around the plant, each of which was done by hand (Table 1). In this activity, the participants used hypothenar eminence and thenar eminence to pick up growing medium for filling the pot by dominant hand. The upper trapezius was also used for transferring the growing medium from the bucket to the pot, which was similar trend with previous activities, filling a container or a tray with growing medium (Table 2 ).

Making moss balls used the upper trapezius, biceps brachialis, flexor carpi ulnaris, brachioradialis, thenar eminence, and hypothenar eminence (Table 2). Making moss balls required gripping and squeezing, the latter of which required more strength than gripping motion.

Removing leaves from plants, sticking cuttings, sowing seed in a tray, and dividing plants mainly required gripping, using the thumb and forefinger. Therefore, the thenar eminence and hypothenar eminence displayed 


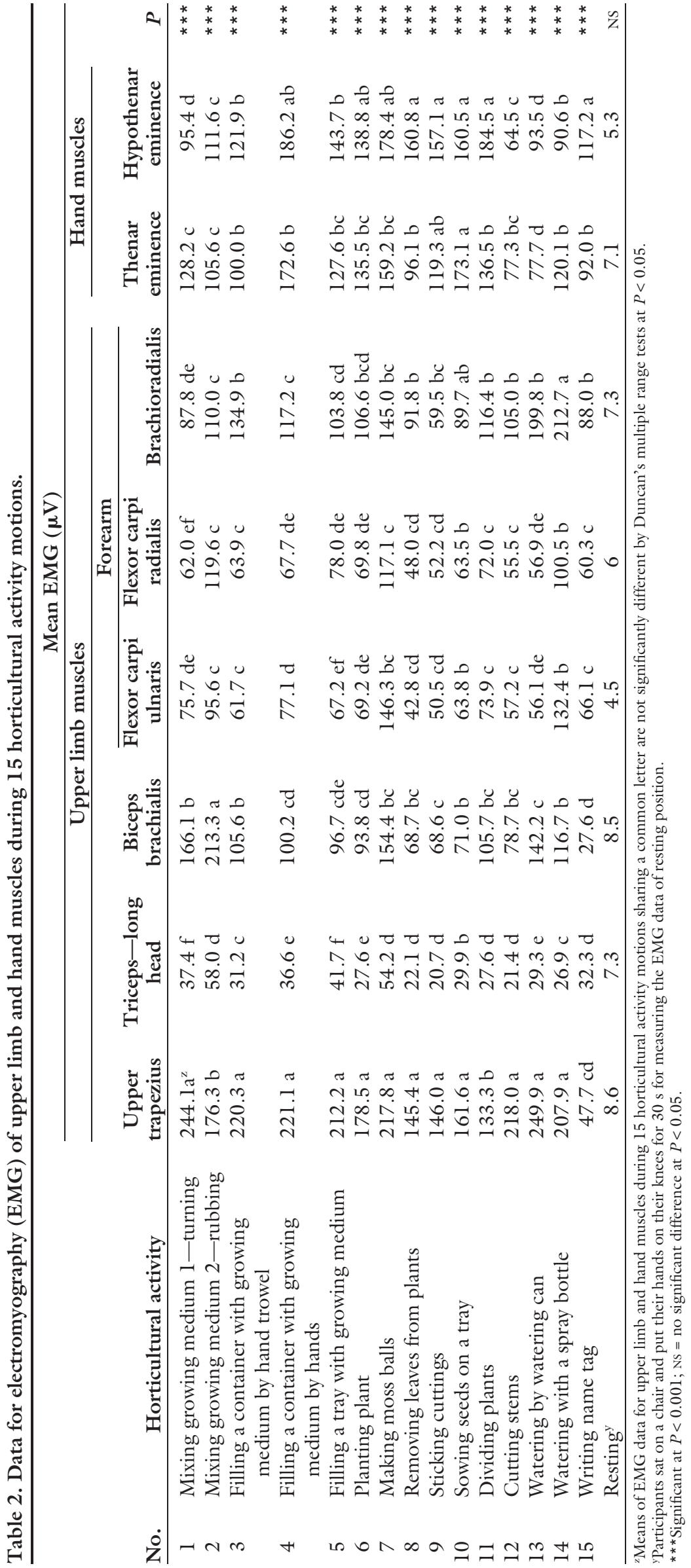

higher EMG values than the upper trapezius (Table 2).

The participants primarily used their upper trapezius and brachioradialis when watering with a watering can and when cutting stems (Table 2). Both activities consisted of weightbearing motions (e.g., holding and squeezing a tool). Watering was the highest weight-bearing activity among all of the activities because of the weight when full $(1.3 \mathrm{~kg}$ ) (Table 1$)$. Spray watering also involves a weightbearing action where $0.5 \mathrm{~kg}$ of spray was lifted and repeatedly squeezed. The upper trapezius, biceps brachialis, flexor carpi ulnaris, flexor carpi radialis, brachioradialis, thenar eminence, and hypothenar eminence were significantly used (Table 2).

Hand muscles (e.g., thenar eminence and hypothenar eminence along with the brachioradialis of the forearm) were used when writing name tags (Table 2 ). The participants gripped a pen and then wrote the names repeatedly, in particular using the thenar eminence.

Given all the results, for almost all activities the primary muscle used was the upper trapezius, an upper limb muscle. The upper trapezius is usually used in most of the activities using neck and arms and appears to be more activated during the activities in sitting than in standing position (Astrand et al., 2003). Six activities-filling a container, planting, making moss balls, removing leaves, sticking cuttings, and sowing seeds-also demonstrated primary use of a hand muscle, the hypothenar eminence. Gardeners had previously been shown to have higher hand function ability (hand strength and pinch force) than nongardeners (Park et al., 2009; Reynolds, 1999). In an observational study, gripping was required in more than $80 \%$ of the gardening tasks tested (e.g., weeding; cleaning tools, hands, or produce; carrying tools; harvesting; watering; cutting flowers or stems; pruning; mowing; deadheading; digging; mulching; and planting plants) (Park and Shoemaker, 2009). The horticultural tasks included gripping or squeezing and would involve using the thenar eminence and hypothenar eminence. Relf (1973) also suggested that gardening activities provide an opportunity to practice grasp and release and flexion of thumb and forefinger. Sowing seeds and watering 
with a spray bottle demonstrated the widest dominant muscle use, with the upper trapezius, brachioradialis, thenar eminence, and hypothenar eminence all primarily involved. This implies that these horticultural activities would be useful for subjects who need to improve the upper limb and hand function. Involvement of the forearm, through the use of the brachioradialis, was demonstrated when using a spray bottle, but the forearm muscles were not dominant for the other activities. This finding that a horticultural activity can specifically improve a certain muscle part of human body is fundamental and useful information and can be applied to developing horticultural therapy program. Consistently low EMG values in most of the horticultural activities were observed in the triceps-long head. This muscle is primarily used in extension motion such as pushing with arms or extending the lower arm, whereas the activities such as lifting objects requires biceps to be active as a flexor and the role of triceps is mostly antagonistic to biceps (Astrand et al., 2003) during the horticultural activities used in this study. Thus, the EMG values of the triceps were generally low during the horticultural activities used in this study. Regarding gender, muscle strength of female is generally known as $\approx 70 \%$ of the male muscle strength (Astrand et al., 2003). However, there was no significant difference for EMG data of the upper limb and hand muscles tested except upper trapezius for the horticultural activities in this study (data not shown). Lee et al. (2012) conducted an EMG analysis for the motions of flower arrangement such as cutting, bending, fixing, and winding to develop a horticultural therapy program for rehabilitation by using flower arrangement movements. Various horticultural activities have been also applied as a treatment in horticultural therapy for physical rehabilitation (Jung, 2005; Kim, 2001), but there is not enough supportive data for the mechanisms on how the various horticultural activities affect the human physical body. The electromyographic analysis of this study provides fundamental and scientific information in the aspect of muscles.

In conclusion, six muscles (i.e., upper trapezius, triceps-long head, biceps brachialis, flexor carpi ulnaris, flexor carpi radialis, and brachioradialis) in the upper limbs and two (i.e., thenar eminence and hypothenar eminence) in the hand were varied when doing a cross section of horticultural activities. Overall, the upper trapezius, thenar eminence, and hypothenar eminence in the hand displayed higher muscle activity than the other muscles measured. Meanwhile, the triceps-long head displayed very low EMG values compared with the other muscles suggesting little link between the activation of triceps, an upper limb muscle, and common indoor horticultural activities. The thenar eminence and hypothenar eminence were the primary muscles used for gripping, which is one of frequented motions used for the horticultural activities. The upper trapezius was primarily used for activities such as transferring, lifting, and weightbearing motions where holding or squeezing was required. To develop scientifically based gardening intervention or horticultural therapy programs for improving physical health or for physical rehabilitation, EMG data for horticultural activities are invaluable.

\section{Literature cited}

Ainsworth, B.E., W.L. Haskell, S.D. Herrmann, N. Meckes, D.R. Bassett, Jr., C. Tudor-Locke, J.L. Greer, J. Vezina, M.C. Whitt-Glover, and A.S. Leon. 2011. 2011 compendium of physical activities: A second update of codes and MET values. Med. Sci. Sports Exerc. 43:1575-1581.

Armstrong, D. 2000. A survey of community gardens in upstate New York: Implications for health promotion and community development. Health Place 6:319-327.

Astrand, P., K. Rodahl, H.A. Dahl, and S.B. Stromme. 2003. Textbook of work physiology: Physiological bases of exercise. 4th ed. Human Kinetics, Champaign, IL.

Bolgla, L.A. and T.L. Uhl. 2007. Reliability of electromyographic normalization methods for evaluating the hip musculature. J. Electromyogr. Kinesiol. 17:102-111.

Chen, T.Y. and M.C. Janke. 2012. Gardening as a potential activity to reduce falls in older adults. J. Aging Phys. Act. 20:15-31.

De Luca, C.J. 1997. The use of surface electromyography in biomechanics. J. Appl. Biomech. 13:135-163.
Fujii, S. and T. Moritani. 2012. Rise rate and timing variability of surface electromyographic activity during rhythmic drumming movements in the world's fastest drummer. J. Electromyogr. Kinesiol. 22:60-66.

Gunn, S.M., A.G. Brooks, R.T. Withers, C.J. Gore, J.L. Plummer, and J. Cormack. 2005. The energy cost of household and garden activities in 55- to 65-year-old males. Eur. J. Appl. Physiol. 94:476486.

Hawkes, D.H., O. Alizadehkhaiyat, A.C. Fisher, G.J. Kemp, M.M. Roebuck, and S.P. Frostick. 2011. Normal shoulder muscular activation and co-ordination during a shoulder elevation task based on activities of daily living: An electromyographic study. J. Orthop. Res. 30:53-60.

Hawkins, J.L., K.J. Thirlaway, K. Backx, and D.A. Clayton. 2011. Allotment gardening and other leisure activities for stress reduction and healthy aging. HortTechnology 21:577-585.

Hong, C.W. and Y.C. Kim. 2009. The evaluation of workload on lower limbs muscles in imbalanced lower limbs postures using EMG for preventing WMSDs. J. Ergon. Soc. Korea 28:81-85.

Jung, S.A. 2005. Effect of the application of horticultural therapy program by phases of psychological adaptation on the psychosomatic rehabilitation in stroke patients. Konkuk Univ., Seoul, Korea, MS Thesis.

Kim, S.Y. 2001. Effect of horticultural therapy on the functional rehabilitation in hemiplegic patients after stroke. Konkuk Univ., Seoul, Korea, MS Thesis.

Lee, S.S., S.A. Park, O.Y. Kwon, J.E. Song, and K.C. Son. 2012. Measuring range of motion and muscle activation of flower arrangement tasks and application for improving upper limb function. Korean J. Hort. Sci. Technol. 30:449-462.

Olivetti, L., K. Schurr, C. Sherrington, G. Wallbank, P. Pamphlett, M.M. Kwan, and R.D. Herbert. 2007. A novel weightbearing strengthening program during rehabilitation of older people is feasible and improves standing up more than a non-weight-bearing strengthening program: A randomised trial. Austral. J. Physiother. 53:147-153.

Park, S.A., K.S. Lee, and K.C. Son. 2011. Determining exercise intensities of gardening tasks as a physical activity using metabolic equivalents in older adults. HortScience 46:1706-1710.

Park, S.A., K.S. Lee, K.C. Son, and C.A. Shoemaker. 2012. Metabolic cost of horticulture activities in older adults. J. Jpn. Soc. Hort. Sci. 81:295-299. 


\section{Research Reports}

Park, S.A. and C.A. Shoemaker. 2009. Observing body position of older adults while gardening for health benefits and risks. Act. Adapt. Aging 33:31-38.

Park, S.A., C.A. Shoemaker, and M.D. Haub. 2008a. Can older gardeners meet the physical activity recommendation through gardening? HortTechnology 18:639-643.

Park, S.A., C.A. Shoemaker, and M.D. Haub. 2008b. A preliminary investigation on exercise intensities of gardening tasks in older adults. Percept. Mot. Skills 107: 974-980.

Park, S.A., C.A. Shoemaker, and M.D. Haub. 2009. Physical and psychological health conditions of older adults classified as gardeners or nongardeners. HortScience 44:206-210.
Relf, D. 1973. Horticulture: A therapeutic tool. J. Rehabil. 39:27-29.

Restuccio, J.P. 1992. Fitness the dynamic gardening way. Balance of Nature Publishing, Cordova, TN

Reynolds, V. 1999. The Green Gym: An evaluation of a pilot project in Sonning Common, Oxfordshire, Rpt. No. 8. Oxford Brookes Univ., Oxford, UK.

Sommerfeld, A.J., T.M. Waliczek, and J.M. Zajicek. 2010. Growing minds: Evaluating the effect of gardening on quality of life and physical activity leve of older adults. Hort Technology 20 : $705-710$

Turner, C.H. and A.G. Robling. 2003. Designing exercise regimens to increase bone strength. Exerc. Sport Sci. Rev. $31: 45-50$
Turner, L.W., M.A. Bass, L. Ting, and B. Brown. 2002. Influence of yard work and weight training on bone mineral density among older U.S. women. J. Women Aging 14:139-149.

Van Den Berg, A.E. and M.H.G. Custers. 2011. Gardening promotes neuroendocrine and affective restoration from stress. J. Health Psychol. 16:3-11.

Waliczek, T.M., J.M. Zajicek, and R.D. Lineberger. 2005. The influence of gardening activities on consumer perceptions of life satisfaction. HortScience 40:13601365 .

Walsh, J.M.E., A.R. Pressman, J.A. Cauley, and W.S. Browner. 2001. Predictors of physical activity in community-dwelling elderly white women. J. Gen. Intern. Med. 16:721-727. 